\title{
L'ago variabile del Marocco: leggenda o realtà?
}

\author{
Manuel Barbera \\ Università degli studi di Torino \\ b.manuel@inrete.it \\ Ilenia Girlando \\ Independent scholar \\ ilenia.girlando@gmail.com
}

\begin{abstract}
In Moroccan Arabic the existence of a variable term depending on the different moments of the day has become an urban legend. That's not the case. This phenomenon finds verification in this context and we attempt to explain it.
\end{abstract}

\section{Keywords}

Urban legends - Moroccan Arabic - chronopathic verbs - needle - Fillmore

Mai tambaya ba shi bata, sai dei asheerinsa ka tonoa

He who asks does not go wrong, but his secret is dug up PROVERBIO HAUSA (MERRICK 1905: 14, N. 238)

\footnotetext{
* Per i rituali scopi di legge va attribuita a M. Barbera la prima metà dell'articolo (§§ o-3), a I. Girlando la seconda metà ( $\left.§ 4_{4.0-4.3}\right)$ e le conclusioni $(\S 5)$ ad entrambi, anche se il lavoro è stato condotto insieme e ambedue si riconoscono parimenti responsabili di tutto il testo. Si ringraziano in particolare Simone Bettega, Riccardo Contini, Franco Crevatin, Luca D’Anna, Fabio Gasparini, Salvatore Granata, Majida Messafi, Valentina Serreli, Salvatore Claudio Sgroi, Mauro Tosco e l'anonimo autore del primo referee, i cui suggerimenti sono stati assai utili.
} 


\section{Lo scenario}

In un passo (di cui inizialmente taceremo l'autore, così come pecetteremo la sua fonte) si racconta:

Digression: while I am on the subject of the ways in which the use of a linguistic expression requires the speaker's awareness of the time of day, I should point out that the traditional greetings in a great many languages are selected according to the time of day, as, for example, English "good morning" and "good afternoon" and the like. The one example of a naming expression whose appropriateness is determined by the time of day in which it is used is an example I have from XY and which I have managed to bring into every lecture I've ever given on the subject of deixis. In Moroccan Arabic there are two words for needle; one of them is used only in the morning, and the other is used during the rest of the day.

L'aspetto è molto quello di una leggenda metropolitana; in questo concorrono l'oralità della fonte e la struttura del dato: variazioni diacroniche sono ovviamente ben note, ma ricorrenti nell'arco della giornata di solito no.

Inoltre, anche in linguistica non è che manchino precedenti famosi: i miti più noti (e spesso ancora, nonostante tutto, risorgenti in forme diverse) sono forse due, ormai vecchi più di un secolo. Il primo è quello dell'esistenza di lingue "primitive" come il Cherokee con i suoi presunti tredici verbi per "lavarsi" (Hill 1952; Scancarelli 1994); e il secondo quello delle parole per "neve" in eskimo (Martin 1986; Pullum 1991b); e non è che ne manchino altri, anzi hanno ispirato addirittura dei libri: almeno Pullum (1991a; Bauer-Trudgill 1998; Wilton 2004).

Inoltre, se posso contribuire con un dato personale (M. Barbera), io l'ho sentito anche riferito alla cruna dell'ago, ma non saprei indicare dove o da chi (l'ho ricercato invano: l'ho "sentito" e basta): è d'altronde tipico delle leggende metropolitane non esserci mai una fonte esatta. L'eziologia è comunque un probabile incrocio del passo sopra citato (che ha buone chances di essere il primum di tutta la vicenda) con la famosa parabola del cammello e della cruna dell'ago,

Et iterum dico vobis | facilius est camelum per foramen acus transire | quam divitem intrare in regnum caelorum

MATTEO, 19.24 (WEBER, GRYSON 2007: 1556A) 
che è anche ripresa nella sura settima del Corano:

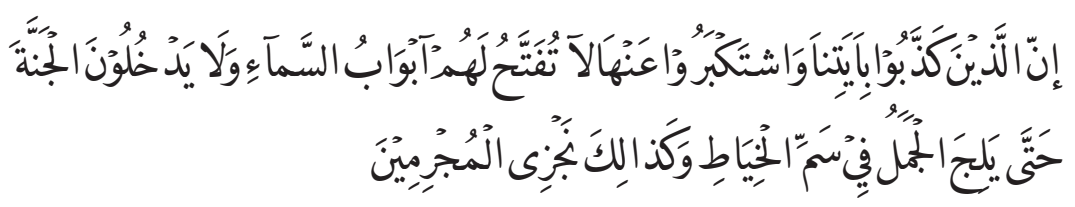

In verità coloro che hanno smentito i Nostri Segni, e superbamente se ne sono allontanati, non saranno spalancate per loro le porte del cielo e non entreranno nel Giardino del Paradiso prima che il cammello sia entrato nella cruna d'un ago: cosi Noi ricompensiamo gli scellerati.

CORANO, ${ }^{1} 7.41$

Tutto bene, dunque? Mah ... Le notorie idiosincrasie dei dialetti maghrebini, consociate alle molte varianti linguistiche autoctone, potrebbero far sorgere qualche perplessità (ma queste potrebbero anzi essere uno dei fattori su cui si appoggia la leggenda), però sono i nomi che finora abbiamo taciuto, ma che ormai è tempo di svelare, a far saltare il castello (indiziario) in aria: il testo è tratto dalle Santa Cruz Lectures di Charles Fillmore (1971), e precisamente dalla Quarta lezione (la prima sulla deissi, a p. $58^{2}$ dell'originario samizdat) e l'XY è nientemeno che Charles Ferguson. Ora l'autorevolezza dei personaggi ${ }^{3}$ è indubbiamente tale da più che insospettire e invita ad ulteriori indagini.

Tanto per iniziare va detto che in arabo classico i termini per indicare "ago" sono principalmente due: hiyāt (خياط; cfr. Wehr 1976: 267b) e ibra (إِّرَّة; cfr. Wehr 1976: 1b s.v. ابرة). Nella sura settima del Corano è utilizzato il termine hiyāt, che deriva dalla radice araba HYṬ ("cucire, rammendare").

In darija (l'arabo marocchino) la situazione è più complessa. Il compendio di varianti dialettali e parlate presenti, infatti, vede il berbero come lingua storicamente autoctona (per dirla con Durand 1994: 5 e sgg.) del Marocco;

1 Testo da Sher 'Alī (2004: 169). Traduzione di Alessandro Bausani (1995: 109), che difatti annota: "chiara eco del passo evangelico Math. XIX, 24" (ibid.: 548). La numerazione del versetto in Sher 'Alī presenta una discrepanza: diamo quella di Bausani, in quanto quella più generalmente invalsa.

2 Se ricostruiamo correttamente la paginazione: ci sono esemplari con numerazioni diverse.

3 E le Santa Cruz Lectures sono forse uno dei testi di linguistica generale più importanti del secondo Novecento. 
con l'arrivo dell'Islam, a partire dal viI secolo, è l'arabo a sovrapporsi alla lingua principale mentre, al termine del XV secolo, con l'approdo degli ebrei in Marocco cacciati dalla Penisola iberica, comincia ad imporsi come lingua della cultura (ibidem), destinando così il berbero e gli altri dialetti marocchini ad essere parlati in àmbiti diversi.

Pertanto le fonti più usuali per la darija dànno un quadro parzialmente differente:

needle: ybra-ibra (f) / ybari—ibari (Abdel-Massih 1982: 424a); aguja: ībra, yibra, bra "aguja, pinchazo (médico)". Pl.: ìbāri, yibrāt. Dim.: ībìra. 2. maxyaț "aguja grande para coser". Pl.: mxāyț. 3. mūri (del reloj). Pl.: mwāra. el-mwāra d el-māgāna "las agujas del reloj" (Moscoso García 2005: 26a);

mexyet pl. mxayet large needle (from a few inches to one or two feet) used for sewing tents, mattresses, certain trappings for beasts of burden, etc.; also used to goad the beasts (Harrell, Sobelman 2004:129 s.v. mexyet); Aguja: ibra (pl. ibari); məftah (pl. mfatəh̆) (Aguadé, Benyahia 2005: 189).

$\mathrm{E}$ anche il quadro che si può ricavare per fasi più antiche è complesso, si veda ad esempio:

AGUJA, ابرة ibra, pl. en át y اباري ibári. ${ }^{4}$ —grande, مفتاحة meftáha, pl. en át.—de enjalmar, مسلّة mesál-la, pl, en át, Cañ.; مخيط májiat o méjiet, pl. موري múrī, pl. موري mejáit.—de reloji._de marear. V. BRÚJULA.-(pez), مخيط ấmiat; عراب ấb. Ojo de la aguja الابرة عين مورين âáīn el-ibra; (الابرة خرم), Cañ.) (Lerchundi 1892: 35a).

In Heath (2002), peraltro, e nel Wortatlas arabischen Dialekte (Behnstedt, Woidich 2012), infine, non è menzione dell'ago, da cucire o meno.

\section{$2 \quad$ Dietro le quinte}

Già da questa bibliografia minima, con la sua polivocità, si evince che forse ci sia sotto qualcosa.

L'antropologo finlandese Edward Westermarck nel suo Ritual and Belief in Morocco ci dice (cit. anche da Aguadé 2010: 278, n. 19) che portava sfortuna menzionare un oggetto tagliente o appuntito:

4 Si è mantenuto inalterato l'originale, con il suo sistema di trascrizione diverso dal moderno. 
There are still other precautions which have to be taken when tents are pitched. Among the Ulâd Bůăzîz the ridgepole of one tent must not be on a line with that of another-l-hommâr ma igâbel l-hommâr; if this rule is not observed, the people inhabiting the tents and the animals which are kept between them will have to suffer. In the same tribe one of the needles (mhait, sing, măhyaț) with which a tent has been sewn must be hung up inside it and left there for three days, it is called 'ázri l-háima, "the bachelor of the tent". So also among the Ait Yúsi and the Ait Sáddĕn the needles (issügy na [Ait Yúsi] or issı̆gy nan [Ait Sáddĕn], sing. issıggy ni) are hung up on the ridge-pole (ahămmar) for three days, or among the former, according to another account, for seven days, $[\ldots]^{5}$

Pointed and sharp objects are often regarded as ill-omened. It is bad fäl to find a nail (Fez, Tangier) or a needle (Tangier) in the street or on the road. [...]

The fear of ill-omened persons, animals, or objects extends to their names, for which euphemistic expressions are often substituted. ${ }^{6}$

WESTERMARCK 1926: II, 26 E 29

E una tale fenomenologia legata agli oggetti taglienti o appuntiti ce la riporta oggi anche D'Anna c.p. (già segnalata in D'Anna 2014: 214 cit. infra).

Siamo pertanto in evidente area di tabù, infatti:

The use of sārūt "key"7 (a word of Berber origin) instead of məftah in many Moroccan dialects is the result of a peculiar semantic shift: maftah (originally "key") being the usual word for "needle" (to avoid ibra), it was necessary to find a new term for "key" and so this Berber loanword has been borrowed.

AGUADÉ 2010: 278

5 Pure qui la trascrizione dei termini è stata riportata fedelmente come nell'originale, ma anche per il motivo che lo stesso Westermarck (1926: I, 85 e sgg.) spiegava: "in rendering Arabic and Berber words and phrases used in Morocco I endeavour to represent them as they are pronounced by the natives, independently of the written Arabic. [...]", e pertanto "a small letter above the line is considered to be pronounced with a reduced sound. [...] The vowel sound of the words are subject to great variations, not only in different localities, but in the same locality, and even in the mouth of the same individual. [...]". Il risultato, quindi, appare oggi (come molte delle trascrizioni usate nell'Ottocento e nel primo Novecento) altamente idiosincratico, ma le intenzioni che lo muovevano sono modernissime.

6 Citando lo studio di Marçais (1906) sull'eufemismo. Più in specifico sulla politeness (ma limitato all'arabo palestinese) cfr. anche Shivtiel (2010), e in generale sulla tabuizzazione cfr. Shivtiel (2009).

7 Sul coinvolgimento delle chiavi in riti ebraici si veda peraltro Cohen (1964: 21, n. 1). 
Che più in generale osserva che "it is well-known that euphemisms play an important role in all languages; North African Arabic dialects are of course not an exception; quite the opposite: in these dialects they are especially frequent and widespread" (Aguadé 2010: 277), cosa che è peraltro ben nota da tempo:

L'euphémisme est universel: est bien développé dans le domaine sémitique, et bien connu chez les juifs et les arabes. Dans l'Afrique du Nord, il est d'un usage très général. On évitera par exemple les mots khâli, khâoui qui signifient vides, déserts: ils sont spécialemant redoutés. Le mot nâr, feu, évoquant l'idée des peines éternelles sera remplacé par 'afta qui veut dire paix.

DOUTTÉ 1909: 365

Doutté prosegue poi per un'altra pagina a riportare interessanti esempi, che non riferiamo tutti per non allontanarci troppo dai nostri scopi.

Per l'ago, comunque, ciò è ad esempio confermato da Chebel, che così compendia Westermarck,

AIGUILLE $(i b r a ̂)$. En raison de la matière dont elle est faite et sourtout parce que sa forme pointue la prédestine à faire du mal, un interdit local louche l'aiguille. Aussi, à l'instar des lames tranchantes et de tout objet contondant, l'aiguille subit un dénigrement amplement compensé, cependant, par les nécessités de l'usage quotidien qui l'imposent au sein de l'attirail de la couturière. Par un procédé euphémistique, l'aiguille à coudre est appelée à Tlemcen (Algérie) mfithâ, litt. "petite clé" (Marçais).

CHEBEL 2001: 23B

incrociandolo anche con Marçais (1906: 435), cit. pure da Aguadé (2010: 277, n. 16), che a sua volta riferiva come in Algeria (Tlemcen) avvenisse una sostituzione di "ago" analoga a quella segnalata da Westermarck in Marocco: $n f i t ̧ h a^{8}$ propriamente "petite clé" veniva usato al posto di yẹ́bra e mefţâh "la grande aguille à coudre" piuttosto che máxyåt (Marçais 1906: 435 procedeva poi a raccontare di altre sostituzioni eufemistiche). Anche D'Anna (2014: 250) ne parla non per il Marocco, riportando (sempre con riferimento prevalentemente a

8 A Tlemcen è a volte avvenuto uno scambio tra la mim e la nun (lo ha documentato Marçais 1902: 22-23, cit. anche in Marçais 1906: 435 n. 1), sicché la parola in realtà è la medesima. 
Marçais 1906: 435) nella fattispecie per l'Algeria " $m f i t h a \hat{a ~ ' L i t t l e ~ k e y ' ~} \rightarrow$ 'Needle", osservando che "the reasons that cause the replacement are not always clear".

Dombay (1800: 39-40; cfr. infra) aggiungeva inoltre ancora altri termini (nove, tra cui il "ferro", segnalazione forse illusoria, se da intendersi come oggetto acuminato, ${ }^{9}$ o comunque problematica) per cui avveniva una sostituzione, ma si trattava più di forme di cortesia che di tabù: "habent etiam Mauri quasdam expressiones, seu vocabula propria, in præsentia regis, vel alicujus personæ, dignitate conspicuæ, usitata" (ibid.: 39).

\section{3}

\section{La variazione temporale}

Le varianti dialettali, come si sarà notato, sono molto diverse anche all'interno di una stessa città o zona e saranno, magari, dovute anche alle influenze di etnie non necessariamente "arabe",10 commistione che in Marocco è particolarmente rilevante.

E questo certo facilita l'innesco del fattore temporale: il terreno è senz'altro fertile e l'area è dunque proficua.

Lo spunto rivelatore, che lega il tabù sull'ago alla scansione della giornata, è riportato ancora una volta dal solito Westermarck:

The broom is euphemistically called măslâha "one that is making [something] good" (Tangier). At Fez, if a person asks another to give or lend or sell to him a big needle, such as is used for the sewing of coarse material, he says méfts ạ "key" (literally "opener"), instead of máhyạt, and if he asks for ordinary sewing needle he says méfts âha instead of yíbra. In the Hiáina

9 Dombay (1800: 40) testualmente recita " المعدن l elmáden. Fodina pro حديد hadid, ferrum". Che pertanto il ferrum non sia da intendere come la usuale metonimia per gladium ma sia proprio il semplice metallo è chiaro non solo dalla scelta del sostituto ('miniera'), ma anche dal termine di partenza (hadìd in Wehr 1976: 16oa è solo il metallo). A meno che il ferro non sia invece proprio semplicemente "ferro" (come, letteralmente, è ben possibile), secondo quanto riportava Doutté (1909: 365): "le mot fer, h'edíd, à cause du caractère magique du fer est volontiers remplacé par ma'aden, mineral", ma la motivazione sarebbe un'altra, su cui torneremo in séguito.

10 L'arabizzazione delle lingue del Nord Africa è stata considerata una vera e propria colonizzazione allo stesso modo di quella successiva francese e inglese negli stessi stati: cfr. ad es. Brugnatelli, Lafkioui (2016). Tra l'altro Westermarck (1926: I, Introduction) notava che, sebbene i Berberi considerassero l'arabo quale lingua superiore alla loro, la rispettassero e si vergognassero della propria, "they do not like the Arabs". 
a máhyạt is in the morning called múftsah and a yíbra müftsáha. Among the Ait Sáddĕn the words täsärutt, which means both sewing needle and key, and iss $\check{g}^{y} n i$, which means big needle, must not be mentioned in the morning, but a sewing needle should be called tälmĕftăht "small opener", and a key and a big needle älmëfțäh, "opener", and at the threshing floor the word issiggy ${ }^{y} i$ must not be used at any time of the day. ${ }^{11}$

WESTERMARCK 1926: II, 28-29

In Morocco, as elsewhere, certain words must be avoided on certain occasions. Thus the Ait Sáddĕn insist that in the morning, or in the presence of a shereef, an earthenware pan (afan or, if small, tëäfant) shall be called úmlil or túmlilt ("white"), and an earthenware kettle (lmáun) túmlitț; and that in the morning a sewing-needle (țäsärutt) shall be called țälmĕftăht ("a small opener"), a big needle (issı̆gyni) älmĕftäh ("opener") [...].

WESTERMARCK 1914: 37 N. 1

La cosa, però, parte ancora più da lontano, almeno dalla fine del Settecento, ${ }^{12}$ ed è attestata per la prima volta probabilmente da Franz Lorenz von Dombay, ${ }^{13}$ "interprete" imperiale a Vienna in età napoleonica:

\section{Tempore matutino dicunt Maurio.}

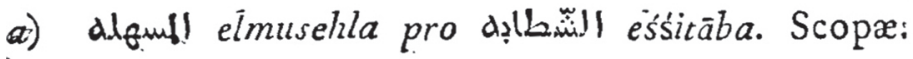 \\ b) dalïill elmiftāha pro o, ll ibra. Acus.

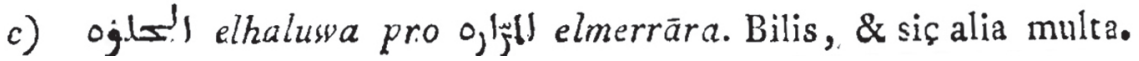 DOMBAY 1800: $40^{14}$}

Complessivamente un insieme, oltre che molto rarefatto, senza dubbio bello eterogeneo: al di là dell'ago (in più di un tipo) e della scopa, cui dandosi parecchio da fare si potrebbe anche trovare qualche tratto in comune, ${ }^{15}$ ci sarebbero pure la bile (nel solo Dombay) e due fogge distinte di pentole di coccio (nel

\footnotetext{
11 Per la trascrizione cfr. la nota 5.

12 Probabilmente la raccolta risalirà al periodo 1783-87 in cui Dombay era "cónsul y traductor imperial en Tánger" (Guerrero 2012: 242).

13 Sul Dombay e sulla sua opera marocchina informa ora Guerrero (2012).

14 Sui limiti delle trascrizioni del Dombay cfr. Guerrero (2012: 243).

15 La natura acuminata dell'uno e dei costituenti dell'altro (cfr. nota seguente): entrambi possono ferire.
} 
solo Westermarck), per cui sembrerebbe non esserci molto da fare (ma vedremo, invece, che una motivazione forse c'è: cfr. oltre). A meno, naturalmente, che non esistano davvero gli alia multa del Dombay, di cui è lecito dubitare.

Quanto alla "scopa", già eśśitāba (che Guerrero 2012: 247 reinterpreta come š-štțāaba), che deriva con molta probabilità dalla radice araba ŠṬB "to cut into slices or stripes; II f. to make an incision" (cfr. Wehr 1976: 471a), è un termine, raro e antico, del dialetto "mauritano"16 sostituito da elmusehla (l-mŭsahhla nella reinterpretazione di Guerrero 2012: 247), altrettanto raro e antico (cfr. müsehlet, Jenisch 1780-1802: IV, 549b ${ }^{17}$ ), dalla radice SHL "to scrape off, peel; [...] to file", vedi ad es. suḥäla "file dust" (cfr. Wehr 1976: 40ob).

Quanto invece all'ago, ne abbiamo già diffusamente trattato; comunque l'elmiftāha (l-məftāḥa ricostruisce Guerrero 2012: 247) del Dombay è dalla radice FTH "to open” (cfr. Wehr 1976: 693ab).

Quanto poi alla "bile", che è forse la più 'fuori serie', elmerrāra (l-marrrāara Guerrero 2012: 247) è dalla radice MRR "to be or become bitter" (cfr. Wehr 1976: 9o1b), e il suo sostituto elhaluwa (l-hălwa, Guerrero 2012: 247) è dalla radice H.LW "to be sweet; to be pleasant, agreeable" (cfr. Wehr 1976: 203ab), evidentemente per antifrasi, secondo quanto riferisce Westermarck che "many animals have euphemistic names. [...] The gall-baldder (märrârd) of an animal is termed hilůwa, "sweet" (Fez, Tangier)" (Westermarck 1926: II, 26-27; cfr. anche Doutté 1909: 365).

Quanto infine alle "pentole di coccio", dal Westermarck si partiva e al Westermarck bisogna ritornare; secondo, infatti, quanto riporta, alcuni utensili da cucina neri o affumicati dal nero della cottura, tra cui per esempio le due pentole di coccio menzionate in precedenza, erano visti come pericolosi:

Sooty kitchen utensils are regarded as dangerous. [...] Among the Ait Sádděn, if a person who sets out on a journey or goes out hunting, or goes to visit a shrine or attend a market, meets a woman carrying an earthenware pan (afan or, if small, tä̈fanț) or a pipkin (lmáun) with the black

16 Conosciuto solo all'edizione teresiana del Meninski (Jenisch 1780-1802: III, 418a), dove il termine è riportato come "settābet a. n.s. dialecto Mauritaniae Scopa. Domb. Addit", specificandone la fonte responsabile per l'addizione: il cerchio è davvero molto stretto! Sia l'edizione originale del Meninski (1680: III, 2814b) che quella teresiana dello Jenisch (1780-1802: III, 418b) hanno peraltro ścetbet tradotto normalente con "ramus palmae virens (ramo verde di palma)" e a similitudine "gladius (i rami di palma sono acuminati)" e aliis "procera puella (ragazza slanciata)".

17 Di nuovo proprio solo dell'edizione teresiana del Meninski, e di nuovo glossato dallo Jenisch, loc. cit., "müseleht. a. n.s. Scopa. Domb. Addit". 
side turned towards him, he returns home. At Tangier a person must not pass between or in front of other persons with a sooty pipkin (qádrar), an earthenware saucepan (tájīn) [...] in his hand.

WESTERMARCK 1926: II, 17-18

Ciò potrebbe probabilmente motivare la scelta di sostituire "afan or, if small, tä̈fant" (ibidem) ${ }^{18}$ con "úmlil or túmlilt ("white")" (Westermarck 1914: 37 n. 1), dalla probabile radice TML, cui pertengono anche tumāla "scum, foam, froth" (cfr. Wehr 1976: 106b), che giustificherebbe (anche senza il ricorso all'antifrasi) la qualifica di white:

[...] if anybody lends to another his máqla made of copper he strews into it some flour, which is good fäl calculated to neutralise its blackness. WESTERMARCK 1926: II, 18

Laddove la farina, per l'appunto bianca, ${ }^{19}$ neutralizzerebbe la blackness dell'utensile.

Oltre a queste unità lessicali delimitate c'è anche notizia che esistesse un uso più generalizzato in questo senso. Scriveva infatti Charles Monchicourt all'inizio del secolo scorso, a proposito di una diversa zona del Maghreb, la Tunisia:

Les indigènes d'El-Agbas (fraction des Sayar des Ouled-Ayar), ceux des Haouachem (Ouled-Mehenna des Majeur) et ceux des Ouled-Sbaa (autour d'Hajeb-el-Aïoun) se refusent à confesser leur tribu quand on les interroge à ce sujet le matin, et ils emploient mille détours pour échapper à la question. Nos protégés s'imaginent en effet que les événements qui marquent le début d'une journée ont une influence sur le reste de

18 Connesso con utfiya "trivet, tripod (in ancient times: any one of the three stones supporting a cooking pot near the fire)", cfr. Wehr (1976: 5a): vale a dire che con questo termine si potrebbe intendere una padella in coccio annerita dalla cottura.

19 Inoltre Campbell Thompson (1908: 29-30) riferisce l'uso di un utensile di colore bianco (white cup) nell'arco temporale della notte all'interno di una formula ritualistica pronunciata per scongiurare l'ira del demone: "to drink water drawn overnight is one of the things (along with sleeping all night in a cemetery or throwing one's nail parings into the street) which cause a man to 'sin against himself'. If a man drinks water at night he exposes himself to the power of Shabriri, the demon of blindness. If he is with some one else, he should say, 'I am thirsty'; but, if alone, he must tap on the lid of the jug and address himself by his own name and the name of his mother, saying, 'Thy mother has bid thee beware of Shabriri, briri, riri, iri, ri, in a white cup'”. 
celle-ci. Tuer un animal venimeux est un heureux présage; dire ou entendre des paroles mauvaises est au contraire d'un fâcheux augure, et ce souci est poussé très loin dans le cas qui nous occupe. L'homme qui évite de s'avouer Gabsi pense au gabs (braise, feu). De même, celui qui redoute de publier sa qualité de Hachmi songe au verbe hachem, qui signifie «tailler en pièces, détruire», et quant aux Ouled-Sbaa, s'ils préfèrent taire leur origine dans les premières heures du jour, c'est parce que leur nom rappelle celui du lion.

MONCHICOURT 1908: 6

Monchicourt non si rendeva ben conto della distinzione tra "éviter dans le langage les paroles pouvant être considérées comme de mauvais augure" (ibid: 5) e variabilità nell'arco della giornata, e la zona non è esattamente la stessa, ma la testimonianza resta.

\section{$4 \quad$ Perché}

Che i fatti esistano, dunque, e che Ferguson e Fillmore non se li siano sognati, a questo punto, è certo; spiegarli, però, è un'altra cosa: "the reasons that cause the replacement are not always clear", diceva giustamente D'Anna (2014: 250), ed è sempre più vero.

In sé, infatti, non è strano che gli oggetti acuminati siano percepiti come 'male' e pertanto siano suscettibili di tabuizzazione e duplicazione terminologica. Ma di solito lo sono in serie, mentre qui pare che quasi solo l'ago ne sia interessato; e il caso delle pentole di coccio che riporta Westermarck non farebbe certo parte della stessa serie, né quello della bile di Dombay. In generale, è vero che è una caratteristica culturale dell'area: "Maghrebi Arabic dialects are particularly rich of euphemistic expressions that replace nouns designing objects or realities thought of as ominous. The reasons behind such processes can be extremely various" (D'Anna 2014: 249). ${ }^{20}$

Ma se questo è vero, appunto, in generale, è poi la scansione temporale della vicenda a rimanere insolita: non si tratta qui delle usuali formule di saluto (riportate peraltro da Fillmore), dietro le quali c'è una spiegazione 'naturale'.

Tutto farebbe pensare che a monte, a rendere conto dei fatti, vi sia una qualche 'storia' tradizionale, magari basata su un aneddoto coranico, un hadìth, un

20 E avevamo visto questo atteggiamento riferito anche per altre zone del Maghreb come l'Algeria o la Tunisia. 
proverbio o un indovinello popolare. Almeno, le rare volte che ciò avviene in altre parti del mondo è quasi sempre così. ${ }^{21}$

Ma quali tali storie siano non è facile a dirsi: ${ }^{22}$ noi non abbiamo trovato traccia alcuna di tutto ciò, nulla di nulla.

Il sospetto a questo punto è che forse la spiegazione normale non sia davvero quella giusta e che la porta cui bussare sia un'altra. E se non un portone, forse una portina potremmo anche lumeggiarla: la mossa però è un poco complessa e richiede più di un ordine di considerazioni da sole interessanti, ma insufficienti.

\subsection{Verbicronopatici ${ }^{23}$}

La prima è l'insolita disponibilità che alcune lingue semitiche sembrano avere per variazioni lessicali legate a diversi momenti della giornata.

Di solito questo avviene soprattutto per verbi generici o di movimento; ed è tanto più visibile quanto una società è 'tradizionale': "indigenous languages reflect the close relationship between people and their natural environment, embodying the complex relationship humans enjoy with landscape and seasons. These connections can be broken when indigenous languages are severed from the ecosystems in which they arose" (Watson, al-Mahri 2017: 87).

È noto infatti che "classical Arabic exhibits a plethora of terms for 'to go' at various times of day and for various purposes; in Modern Standard Arabic, 'to go' is predominantly expressed with the cover term dahaba with an adverbial phrase to express time of day or manner of moving" (Watson, al-Mahri 2017: $88-89) .{ }^{24}$ Tutte queste forme, in effetti, sono state soppiantate, qual più qual

21 Riferiva ad esempio Franco Crevatin (c.p. a M. Barbera) che nella sua nativa Istria di una persona morta a mezzogiorno si diceva "che ha lasciato un cucchiaio" e di una morta la sera "che ha lasciato una forchetta"; apparentemente ciò è inspiegabile, ma diventa affatto naturale se si fa riferimento alla storia alimentare del luogo, per cui il pasto di mezzogiorno (quando v'era) consisteva solo in una minestra, mentre quello serale era più solido.

22 Né l'eterogeneità del gruppo dei termini sottoposti a questo curioso 'tabù orario', tra l'altro, aiuta: infatti abbiamo scoperto che non sarebbe solo l'ago a essere coinvolto.

23 L'etichetta suona un poco scherzosa; forse il neologismo cronometablematicità (e derivati) sarebbe più adeguato, ma, seppur formato secondo schemi ben consolidati e collaudati, è francamente sgraziato (ricorda un poco il pragmaticism coniato da Peirce, nella dichiarata convinzione che fosse talmente brutto che nessun altro lo avrebbe voluto usare).

24 Già Lombard (1936: 641-42)—che dipendeva dall'orientalista svedese Henrik Samuel Nyberg (1889-1974) - forniva una buona lista, osservando che „diese Sprache besitzt eine ganze Reihe von Verben, die einem Zustand oder ein Handlung als mit einem bestimmten Teil des Tages verbunden bezeichen". 
meno, da ذهب dahaba (Wehr 1976: 313a) associato agli avverbi di tempo che scandiscono i momenti della giornata cui ci si riferisce.

Inoltre in arabo standard moderno ci sono ancora verbi per "diventare" o "trovarsi in uno stato o condizione" che cambiano a seconda dell'ora del giorno, mentre per quanto riguarda i verbi di movimento e nello specifico il verbo "andare" nell'arabo moderno standard è ormai di uso comune indicare i vari momenti della giornata tramite l'uso di avverbi di tempo, a differenza del classico e ancora di molti dialetti arabi in cui permane ed è facilmente riscontrabile l'uso del verbo di movimento "to go/andare" differente a seconda dell'ora del giorno.

Tra i verbi per "diventare" o "trovarsi in uno stato o condizione" variabili, ovأُشى vero che cambiano a seconda dell'ora del giorno, le forme più note sono amsā Un esempio può essere: ?amsaytu marị̂nan wa-șabaḥtu bi-xayr, ossia "ieri sera ero malato e stamattina sto bene". ${ }^{26}$

Analogamente, ci potrebbe essere anche il verbo samara سمر letteralmente "conversare durante la sera o durante la notte" (da samura سمر "essere o diventare buio; essere o diventare bruno"), che si usa al posto del verbo conversare con la scansione del tempo 'notte.' ${ }^{27}$

25 Ringraziamo dell'osservazione e dell'esempio Luca D'Anna. Propriamente صبc șabaha è "dare da bere a qualcuno una bevanda alla mattina / dare a qualcuno la colazione" (Traini 1993: 721), infatti aṣbaḥa vale "farsi mattina / essere mattina / svegliarsi / divenire chiaro" e subh è "la preghiera del mattino prima delle sette / la preghiera dell'alba / il crepuscolo del mattino", che in Marocco è sobh (dato ricavato dalle interviste di cui sotto).

26 Il verbo دأمسى amsā vuol dire lett. "entrare nella sera" (si sostituisce a دخل في المساء, sfr. Baalbaki 2006: 169, dove دخل vale "entrare" e مساء vuol dire "sera"): scandisce quindi un momento della giornata e allo stesso tempo uno stato o condizione (cioè indica uno stato / condizione: marị̣an "malato-ACC"); e similmente è per șabaḥtu (perf. di șabaḥa)

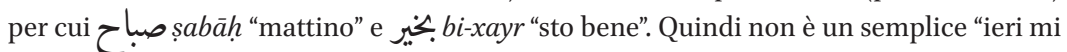
sono coricato malato e oggi mi sono svegliato che sto bene", come pure si sarebbe tentati di interpretare, ma proprio "ieri sera ero malato e stamattina sto bene" con essere (nello stato $X$ ) espresso da due predicati diversi a seconda del momento della giornata.

27 Si sostituisce a verbo hadata "intrattenersi con qualcuno a parlare" e laylan "sera / notte". Da samara ritroviamo "colui / colei che conversa di notte; compagno/a di conversazioni serali / notturne” (samìr, masch. e samīra femm.), ad indicare un'azione che avviene in un dato momento della giornata, che in questo caso corrisponde alla sera / notte. 
Oltre al cُ أصضح aṣaha "essere di mattina" (Traini 1993: 721), "to be, become غد ا gadā "partire di mattina, venire di mattina, essere di mattina presto" (Traini 1993: 1012), "to go (away), leave, come, do, or be, early in the morning" (Wehr 1976: 666b). ${ }^{29}$

Entrambi questi verbi possono voler dire "diventare", ma hanno anche i valori di cui sopra; inoltre esiste un altro verbo per "diventare",

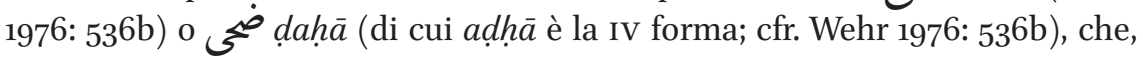
sebbene non abbia significati relativi all'ora del giorno, viene comunque da una radice che ha a che fare con il mattino (cfr. ضصوة dahwa "forenoon, morning", Wehr 1976: 536b).

A questi si può aggiungere (sempre da Lombard 1936: 641) ظقّalla (cfr. Wehr 1976: 581b) "zur Zeit des Tages werden od. sein od. forsetzen (etwas zu Tun)", ب bāta ${ }^{30}$ (cfr. Wehr 1976: 84b) "in die Nacht eintreten (od. die Nacht zubringen), indem man sich irgendwo befindet od. etwas macht”, أسخفر asfara (IV forma di safara) (cfr. Wehr 1976: 412b) "bei Tagesanbruch sein".

Tra i verbi per "andare" (sempre da Lombard 1936: 641): أ غ ġadä (cfr. Wehr 1976: 666b) "am Frühen Morgen kommen, gehen, tun od. Sein” e إ rāḥa (cfr. Wehr 1976: 364a) "am Abend gehen, tun od. sein".

Il fenomeno non è poi così raro come sembra ed è stato segnalato (cfr. Coseriu 1977 con bibliografia; il punto di partenza è comunque Lombard 1936) anche in area ispanica: cfr. spagnolo amanecer e anocher, portoghese amanhecer e anoitecer con, oltre al valore solito (impersonale: "unpersönnlich", sensu; Lombard 1936: 637 n. 1) di "far giorno o notte", anche quello (personale: "persönnlich", sensu; ibid.: 637 e passim) di "den Einbruch des Tages bzw. den Einbruch der Nacht erleben" (ibid.: 637 per il solo spagnolo; riprodotto anche in Coseriu 1977: 40), come negli esempi "yo amanecí en Madrid y anochecí en Toledo „ich befand mich am Morgen in M. und an Abend (desselbenTages) in T.“, amanecí con plata y anochecí sin blanca „me levaté con dinero y me acosté sin el“" (Lombard 1936: 637), laddove i corrispondenti verbi catalani amanèixer e vesprejar hanno solo i valori impersonali delle altre lingue romanze centrali

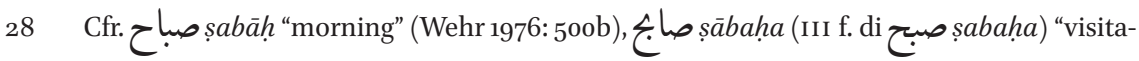
re qualcuno di mattina / incontrare qualcuno di mattina" (Traini 1993: 721).

29 Per cui è facile il riscontro ad esempio con غد gadāh "early morning" (Wehr 1976: 666b) e غد gad

30 "Auch andereStammformen dieses Verbums, wie dieII., dieX., haben "Nachtbedeutungen"” (Lombard 1936: 641). 
(ibid.: 638); in spagnolo, peraltro, la cosa è nota per tutto l'arco della sua storia, dalle origini al latino-americano. ${ }^{31}$

A partire da Lombard (1936) in tali usi si è voluto ravvisare degli "arabismos أصبح semanticos" (Coseriu 1977:40) calcati sugli usi personali della coppia așbaḥa: أمُصى amsā; la cosa è stata variamente discussa, soprattutto in sede di storia della lingua spagnola (gli estremi sono riferiti in Coseriu 1977: 40-43), ma la tesi araba (nonostante gli scettici come Leo Spitzer non siano mancati) è prevalente fino al contributo di Coseriu, che argomenta invece a favore di una origine interna, romanza, della costruzione.

Centrali nell'argomentazione sono i dati del romeno, che conosce solo per uno dei verbi, quello relativo alla notte, un uso personale: a însera. Tali usi erano ben noti (e documentati) già dal Lombard (1936: 638), che anzi era un "excelente conocedor del rumano" (Coseriu 1977:45), ma erano da lui sganciati dal romanzo e dall'arabo e ricondotti ad un generico balcanismo, dato che, forte di Sandfeld (1930: 210-11), li conosceva anche per bulgaro, albanese e neogreco. Coseriu dimostra efficacemente la seriorità dei dati greci e trova qualche spia (ma non più di quelle) in latino, ipotizzando che il primum del balcanismo possa essere romanzo, e che quindi la concordanza romeno-iberica sia valida e la coincidenza con l'arabo vada scartata.

In effetti, dati analoghi si possono trovare anche in un'altra zona della Romania, cioè in Sicilia. Qui, infatti, si può citare il siciliano orientale (Raffadali, Agrigento; dobbiamo l'esempio ancora a L. D’Anna) scurà bbonu e agghiurnà malatu "si è coricato di sera buono e si è alzato la mattina ammalato"; ${ }^{32}$ la reggenza personale di verbi atmosferici non è tuttavia fenomeno pansiciliano: a Catania, ad es., informa S.C. Sgroi, si avrebbe solo agghiunnari con soggetto personale (agghiunnai bbonu "mi sono alzato bene di mattina") ma scurau vorrebbe unicamente dire "ha fatto buio"; e dall'altro lato dell'isola le cose vanno anche peggio, ad es. in liparoto, secondo una consulenza di S. Granata, native speaker eoliano, tanto scurari che agghiurnari si riferiscono rigorosamente alle condizioni atmosferiche (la frase in questione diventerebbe un 'normale' $m i$ curcaiu buonu e abbrisciu malatu). In Sicilia, ossia, si ritroverebbero tutte le situazioni altrimenti attestate nel continente: entrambi i verbi (agrigentino e spagnolo), solo quello notturno (catanese e rumeno) e nessuno (liparoto e catalano).

31 Lombard può citare un esempio della metà del XIII secolo ¿Non veedes las yerbas floridas que amanescen verdes y anochescen secas? e uno della Colombia obscurecí llorando "am Abend weite ich" (Lombard 1936: 639).

32 Che potrebbe, non a caso, ricordare Pirandello. 
I dati siciliani possono, peraltro, confermare o non confermare l'ipotesi di Coseriu: la tesi romanza è ingegnosa, certo, ma cozza contro lo scoglio delle mancate attestazioni in latino; e in tutte le tre zone dove il fenomeno è attestato, anche l'influenza araba è ugualmente attestata. Paradossalmente la impeccabile dimostrazione di Coseriu della natura tardiva delle attestazioni greche potrebbe ritorcerglisi contro, ${ }^{33}$ e forse sarebbe dirimente uno studio delle prime attestazioni in albanese e in bulgaro: ${ }^{34}$ per ora, in verità, non è facile decidersi.

Come che le cose stiano in àmbito romanzo, in campo semitico l'originaria cronopaticità polivoca per "andare" è tuttora ampiamente diffusa (anche se pure lì in regresso) soprattutto nelle lingue neosudarabiche, dove, non a caso, il 'passaggio alla modernità' è abbastanza recente: "the MSAL have several verbs to describe going and coming according to the time of departure. In Central Omani Mehri, traditional verbs of going are: $\dot{g} s \bar{m} m$ [dawn to c. 7am], ahawgar [midday], šūgūś [mid-afternoon], śôfak [just before sunset], abōṣar [twilight, early evening], $b \bar{a} r$ [night]. In western Omani Mehri, hakrawr originally refers to going in the heat of the day" (Watson, al-Mahri 2017: 94); "traditional Omani Mehri verbs of coming are: šaghūm [early morning], ḳhēb [around midday], watxaf [afternoon to sunset], twuh [night]. Today, watxaf remains in common

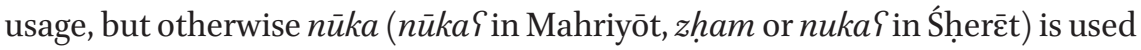
to indicate coming at any time of day together with a time adverbial" (ibidem).

Perché questa generale tendenza (non connessa ad alcuna particolare manifestazione di tabuizzazione o eufemismo) si sia così affermata è poi un'altra questione. Qui non sembra trattarsi di tabuizzazione quanto di codifica più efficiente dell'informazione, però (per evitare di ricadere nel mito antropologico della 'naturalità' delle società 'primitive') un possibile tentativo di spiegazione consisterebbe nel fare comunque appello alla mentalità magico-religiosa, almeno per rinforzare e generalizzare fenomeni per la cui insorgenza isolata basterebbe forse qualche versione ad hoc della consueta arbitrarietà del linguaggio. ${ }^{35}$

33 Meno quella della simmetria col latino (Coseriu 1977: 54-56), invero rimarchevole.

34 Studio che potrebbe intraprendere convincentemente probabilmente solo uno storico delle lingue in questione.

35 Nel caso del siciliano, invece, se fossero indipendenti, sembrerebbero più fenomeni legati alla 'difficoltà' valenziale dei verbi atmosferici, senza escludere le possibili connessioni arabe o romanze di cui si è detto. 


\subsection{La tabuizzazione}

La tabuizzazione e la sostituzione eufemistica, come abbiamo visto, sono elementi caratterizzanti tutto il Nordafrica. Quello che avevamo lamentato è l'apparente asistematicità del fenomeno, che per gli oggetti acuminati è spiegabilissimo ma per altri meno.

In realtà, quasi tutti gli oggetti fino ad ora studiati e ricercati e scoperti e oggetto di uno scambio in senso terminologico (come l'ago, la scopa, il pentolame in terracotta, la bile ...) sebbene apparentemente non legati' tra loro, lo potrebbero diventare se consideriamo quale àmbito di appartenenza quello della 'magia' che è molto più diffusa in Nordafrica di quanto possiamo immaginare ed è legata ad una tradizione più antica dell'Islam stesso. ${ }^{36}$ L'importanza della magia e soprattutto la diffusione della magia nera in Marocco è stata, d'altra parte, ancora recentemente confermata da alcune interviste condotte da I. Girlando ${ }^{37}$ all'inizio del 2017.

La magia, inoltre, permetterebbe di recuperare l'esempio del 'ferro' ${ }^{38}$ sensu Doutté (1909: 365; cfr. supra), che ne fa esplicitamente menzione come spiegazione, riportando anche casi di preterizione non facilmente equivocabili come "parler des puissances mauvaises enfin est dangereux: on ne parle pas du diable sans dire mentalement: 'Je me réfugie, etc ...' (Doutté 1909: 366).

L'uso di aghi, scodelle in terracotta, sacchettini, henna, foglietti con scritture, erbe, ecc. per scopi magici è frequente in Marocco e probabilmente in gran parte del Nord Africa. La spiegazione di solito data è che l'ago in particolare è uno strumento importante in magia perché chiude come sigillo i sacchettini destinati ad un incanto verso una determinata persona (l'incanto in cui si usa tale strumento è in genere un maleficio, una 'fattura' per far del male a

36 Ma che all'islamismo è stata ricondotta e assimilata (non diversamente da molte festività cristiane, ad es. il Natale, o leggende agiografiche che sono state 'cristianizzate' ma hanno origini pagane).

37 La fonte principale è una ragazza di 26 anni la cui famiglia è originaria della provincia di Khourigba in Marocco, figlia di immigrati e nata in Italia, dove è anche stata scolarizzata (materna a Cagliari, elementari e medie a Cavour, e superiori-ragioneria—a Saluzzo). Che informazioni preziose e 'arcaiche' siano a volte conservate nelle comunità all'estero piuttosto che in patria è circostanza abbastanza normale (basti pensare ai piemontesi o ai friulani in Argentina) e, tutto sommato, corrisponde bene alla legge bartoliana delle aree laterali.

38 Usi 'magici' del ferro, ancorché spesso di segno opposto, sono peraltro ben noti al di fuori dell'arabo: "peut-être faut-il rappeller [...] la croyance trés gènèrale chez divers peuples que le fer constitue une protection contre les démons et les esprits", Cohen (1964: 21 n. 1, con bibliografia e riferimenti all'ebraico e al berbero). 
qualcuno). ${ }^{39}$ Nessuno strumento sigilla come l'ago, ${ }^{40}$ né colla né altro, e quindi l'importanza che ha all'interno di questo tabù è fondamentale.

La terracotta è peraltro un materiale considerato 'primario' dagli arabi marocchini sin dall'antichità perché è di terracotta che è fatto il țājin (طاجن,cfr. Wehr 1976: 554a; in darija propriamente si ha tažin, Aguadé, Benyahia 2005: 151), il piatto in cui viene cotta una pietanza di carne in umido, che prende appunto il nome dal piatto stesso (la parola ha origine berbera).

Nelle nostre interviste non è mai stato detto direttamente che il termine ago, o qualsiasi altro dei termini che abbiamo menzionato, sia stato modificato come abbiamo visto, ma è stato ribadito che episodi simili a questo, in cui non si pronunciano alcune parole mentre se ne usano altre, siano da ricondurre con molta probabilità ad un discorso di tabuizzazione strettamente legato alla religione e alle credenze di carattere religioso e magico.

\subsection{Il legame tra cronopaticità e tabù}

Le due sfere, comunque, per quanto culturalmente vicine e concomitanti, restano tra loro non connesse; in altri termini, come abbiamo osservato, i fatti riferiti da Westermarck riguardo l'erezione delle tende potrebbero essere addotti a giustificare la scelta dell'ago, ma non la sua variabilità temporale.

39 Un'altra pratica connessa all'ago (ben nota in àmbito ebraico, ma di cui una variante giunge anche ad un hadìth, cfr. Juynboll 2007: 199, e che, in diverse forme, è largamente diffusa anche altrove, cfr. ad es. per il malese quanto riportava Skeat 1900: 569-70) è inoltre quella di conficcarlo in statuette di cera o di argilla, adeguatamente etichettate, che fungano, per il consueto principio di simpatia, da 'sostituto' della persona da colpire (cfr. ad es. Campbell Thomson 1908: 144-45). In àmbito ebraico, un esempio di sortilegio di questo tipo, in cui l'ago entra nel rituale magico di un incantesimo d'amore, è quello riferito da Trachtenberg: "take virgin wax and make a female figure, with the sex organs clearly delineated, and with the features of the person you have in mind. Write on the breast-, daughter of - [father's name] and-, daughter of-[mother's name], and on the back between the shoulders write the same, and say over it, 'May it be Thy will, O Lord, that $\mathrm{N}$ daughter of $\mathrm{N}$ burn with a mighty passion for me.' Then bury the figure, and cover it carefully so that its limbs are not broken, and leave it thus for twenty-four hours. Then bury it under the eaves, being careful that no one witnesses your acts, and cover it with a stone so that it doesn't break. When you disinter it, dip it carefully in water three times, so that it is washed clean, once in the name of Michael, again in the name of Gabriel, and the third time in the name of Raphael, and immerse it in some urine. Then dry it, and when you wish to arouse passion in her, pierce the heart of the image with a new needle, in that spot where it will cause most pain. So will she daily experience now this pain, now that" (Trachtenberg 1939: 125-26).

Così almeno si dice nell'intervista che abbiamo raccolto. 
E invece una connessione, anche se forse non delle più evidenti, si potrebbe ravvisare proprio in quella dimensione magica che abbiamo delineato.

Tali cambiamenti, si è detto, tendono ad organizzarsi in serie, ma dell'insieme di termini di cui abbiamo trovato traccia abbiamo più volte rimarcato l'eterogeneità: la caratteristica più inclusiva, quella di appartenere alla sfera del casalingo (ago, scopa, pentolame) o, tradizionalmente, del femminile lascia comunque fuori la bile. L'unica caratteristica comune pare proprio l'area del magico, ${ }^{41}$ il ricorso alla quale è certo pericoloso, ma, come diceva Sherlock Holmes nella Adventure of the Beryl Coronet, "when you have excluded the impossible, whatever remains, however improbable, must be the truth" (The Adventures of Sherlock Holmes, 1892, in Conan Doyle 1927: 315). Inoltre anche la pratica 'superstiziosa' riferita da Monchicourt (1908) per la Tunisia vi si accorderebbe.

E poi: sono caratteristiche proprio del magico le scansioni temporali. Per esempio, riporta Cohen (1964: 99) il curioso caso di non scopare di notte per non "svegliare i ginn": "balayer la nuit est dangereux, 'cela dérange les žnūn'. Toutes ce conduites ne relèvent pas de la pure superstition aux yeux du sujet. Elles se rattachent plus ou moins obscurément à des croyances et des pratiques religieuses". Da qui la conferma dell'importanza degli stessi oggetti domestici menzionati in precedenza, la scopa in questo caso specifico, in relazione con la scansione temporale e il conseguente processo eufemistico: "parmi les objets domestiques, il en est un qui a une influence particulièrement redoutable: le balai. Tunis juif ne le désign que par le nom euphémistique de mṣal(!) $h a$ (litt. "celle qui arrange, qui met la paix"), mai peu de personnes ont encore conscience de l'étymologie du mot" (Cohen $1964: 100){ }^{42}$

Già la preghiera islamica va svolta cinque volte al giorno, e ha in effetti nomi diversi a seconda del momento della giornata: i vari momenti della giornata, anzi, sono importantissimi per loro, come lo è la notte, che ha valenza negativa

41 Certo, i riferimenti alla bile nella pratica magica non sono molti e il principale è di nuovo nel Westermarck: « the gall-bladder of the sacrified animals is commonly hung up inside the house or tent, often over the fire-place; there is much baraka in it. In many tribes mothers, for the purpose of weaning their babies, rub their breasts with it so as to give them a bitter taste (Ulâd Bůăzîz, Ḥiáina, Ait Nder, Ait Waráin). [...] Among the Aț Ubáhuti both the gall and the urinary bladder are sospended from the front pole of the tent, and are left there for an indefinite time » (Westermarck, 1926, vol. 2 pp. 126-127), appoggiato anche dal Doutté: «le fiel, mirâra, est souvente appelé h'eloua, doucer, sucrerie » (Doutté 1909, p. 365).

42 "Les tabu linguistiques et les euphémismes sont très fréquents dans le parler des Juifs de Tunis. [...]" (Cohen 1964: 100 n. 3). 
estrema e allo stesso tempo positiva a seconda del periodo, se sacro o meno. ${ }^{43}$ Qui la connessione è con il 'magico': qualsiasi parola che 'richiami il demonio', infatti, non può e non deve essere pronunciata la sera, nello specifico, dopo la terza preghiera della giornata che viene eseguita prima del tramonto. ${ }^{44}$

Definire e scandire, oltre ad essere propri della lingua araba, lo sono anche per la magia.

\section{5}

\section{Conclusioni}

Ferguson avrà colto gli ultimi resti di una tradizione di cui abbiamo ravvisato tracce fin dalla fine del Settecento, e che Westermarck ad inizio Novecento aveva trovato ancora vitale. I dati di Ferguson probabilmente non li sapremo mai, dato che (almeno a quel che ci consta) non sono stati pubblicati, ${ }^{45}$ anche se senz'altro l'humus cui attingeva è quello descritto nei paragrafi due e tre; ma che siano realmente esistiti non è più lecito dubitarne, per quanto poi la cosa abbia subito le consuete fenomenologie e vicende delle leggende metropolitane. Anzi, abbiamo fin potuto abbozzarne un parziale tentativo di spiegazione,

43 La notte ha un'importanza notevole in quanto durante questo periodo sacro del Ramadan i credenti 'tornano in vita': è il momento più atteso della giornata perché si torna a vivere. Diversamente, al di fuori del Ramadan, la notte diventa un 'pericolo', ma comunque degna di attenzione e importanza sebbene per altri motivi. È credenza (le recenti inchieste citate confermano come questa disposizione sia ancora presente in strati tradizionali della società) che con l'arrivo della sera tutte le anime vengano liberate e, nel loro vagare, si impossessino di chi si predispone al loro arrivo. Alcune parole diventano tabù e possono essere pronunciate solo se accompagnate dal nome di Allāh oppure non pronunciate affatto. Durante il mese del Ramadan si è liberi di pronunciare qualsiasi cosa in qualsiasi ora del giorno perché le entità negative, che solitamente si liberano prima del tramonto, rimangono 'incatenate' dal grande sacrificio del digiuno giornaliero, o presumibilmente tale, operato dai credenti. Un uomo vittima di un maleficio durante il mese del Ramadan è libero e torna a stare bene, mentre al termine del periodo sacro torna nella sua condizione di prigioniero e di sofferente.

ساحرة e sahhāara e sāhira "sorceress, whitch", sono infatti collegate a سحّارة sahar "time before daybreak, early morning, dawn" (Wehr 1976: 400a).

45 Inoltre potrebbe anche darsi che siano secondari. Infatti, secondo nota il nostro reviewer: "i dati di Ferguson [...] sono forse stati attinti dal solito Westermarck, di cui F. aveva analizzato (1952) la raccolta di proverbi marocchini, apprezzandone il pionieristico tentativo di descrizione contestuale (1930): è immaginabile che F., più esperto di arabo orientale che maghrebino, abbia voluto leggere anche l'opera etnografica maggiore di W. sul Marocco, facendo tesoro, almeno per la sua riflessione di metodo e per la discussione orale se non nei suoi scritti, dei fenomeni etno- e sociolinguistici salienti che vi aveva trovato". 
in cui gioca un ruolo centrale la sfera del magico; se tra i vari oggetti che vi sono relati (apparentemente irrelati, ma tutti collegati alla sfera della magia) poi è proprio l'ago ad essere il più noto sarà dovuto, oltre che alla sua importanza rituale, anche al suo ruolo, riferito dal Westermarck, nella costruzione delle tende, attività indubbiamente centrale in società tradizionali di questo tipo. Con ciò non sappiamo se davvero il segreto sia stato del tutto dissotterato, come voleva il proverbio hausa in epigrafe, ma possiamo almeno sperare in una parziale esumazione.

Si imporrebbero, a questo punto, forse nuove indagini dialettali, ma è perlomeno dubbio che di una cosa tanto legata alla 'cultura' ad un secolo di distanza, con tutti i cambiamenti che si sono verificati, ve ne siano ancora tracce rilevanti, soprattutto se pensiamo che tale cultura è stata relegata, con ogni verosimiglianza, ad una trasmissione esclusivamente di tipo orale. I dati etnografici recentemente raccolti dalla coautrice potrebbero però fare ancora un poco sperare.

\section{Riferimenti bibliografici}

Abdel-Massih, Ernest T. (1982) An Introduction to Moroccan Arabic. Revised edition (Center for Near Eastern and North African Studies). University of Michigan.

Aguadé, Jordi (2010) The Word for "Nine" in Moroccan Arabic and Other Euphemisms Related to Numbers. Jerusalem Studies in Arabic and Islam 37, 275-82.

Aguadé, Jordi, Laila Benyahia (2005) Diccionario árabe marroquí: árabe marroquíespañol / español-árabe marroquí. Cádiz.

Baalbaki, Rohi (2006) Al-Mawrid. A Modern Arabic-English Dictionary. Beirut. [1992 ${ }^{1}$, $\left.1995^{7}\right]$.

Badawi, Elsaid M., Muhammad Abdel Haleem (2008) Arabic-English Dictionary of Qur'anic Usage (Handbuch der Orientalistik. Abteilung 1. Der Nahe und Mittlere Osten 85). Leiden-Boston.

Bauer, Laurie, Peter Trudgill eds. (1998) Language Myths. London.

Bausani, Alessandro a c. (1995) Il Corano. Milano.

Behnstedt, Peter, Manfred Woidich (2012) Wortatlas der arabischen Dialekte. Band II: Materielle Kultur. Leiden-Boston.

Brugnatelli, Vermondo, Mena Lafkioui (2016) La linguistica e sociolinguistica berbera in Italia: il difficile compito di colmare una lacuna epistemologica. $A I \Omega N$ n.s. 5 , 39-66.

Campbell Thompson, R[eginald] (1908) Semitic Magic. Its Origins and Development. London. 
Chebel, Malek (2001) Dictionnaire des symboles musulmans. Rites, mystique et civilisation. Paris.

Cohen, David (1964) Le parler arabe des juifs de Tunisi. Textes et documents linguistiques et ethnographiques (École pratique des hautes études - Sorbonne. Sixième section: Sciences économiques et sociales. Études juives 7). Paris-Le Haye.

Conan Doyle, Sir Arthur (1927) The Complete Sherlock Holmes. With a Preface by Christopher Morley. New York-London-Toronto-Sidney-Auckland. [19271].

Coseriu, Eugenio (1977) ¿Arabismos o romanismos?, in Estudios de lingüística románica (Biblioteca románica hispánica: Estudios y ensayos 269), 40-69. Madrid. [origin. in Nueva revista de filología hispánica 15 (1961), 4-22].

D’Anna, Luca (2014) Aspects of Verbal Politeness in Maghrebi Arabic Dialects (Tesi di dottorato). Napoli.

Dombay, Francisci (1800) Grammatica linguae Mauro-Arabicae juxta vernaculi idiomatis usum. Accessit vocabularium Latino-Mauro-Arabicum, opera et studio Francisci de Dombay [Franz Lorenz von Dombay]. Vindobonae, apud Camesina.

Doutté, Edmond (1909) Magie \& religion dans l'Afrique du nord. Alger. [Réimpression photomécanique, Paris 1984; poi Bruxelles 2013].

Durand, Olivier (1994) Profilo di arabo marocchino. Roma.

Fillmore, Charles J. (1971) Santa Cruz Lectures on Deixis 1971. Bloomington. [rist. in Lectures on Deixis (CSLI lecture notes 65), Stanford (CA), 1997].

Guerrero, Jairo (2012) La gramática de árabe marroquí del austríaco Franz von Dombay, in Ana Agud (a c.), Séptimo centenario de los estudios orientales en Salamanca (Estudios filológicos 337), 241-49. Salamanca.

Harrell, Richard S[lade], Harvey Sobelman eds. (2004) A Dictionary of Moroccan Arabic: Moroccan-English / English-Moroccan. Washington. [First editions: A Dictionary of Moroccan Arabic: Moroccan-English, ed. by R.S. Harrell, Washington 1966; A Dictionary of Moroccan Arabic: English-Moroccan, ed. by H. Sobelman, Washington 1963].

Heath, Jeffrey (2002) Jewish and Muslims Dialects of Moroccan Arabic. London.

Hill, Archibald A. (1952) A Note on Primitive Languages. International Journal of American Linguistics 17, 172-77.

Jenisch, Bernhard Freiherr von (1780-1802) Francisci a Mesgnien Meninski Lexicon Arabico-Persico-Turcicum, adiecta ad singulas voces et phrases significatione Latina, ad usitatiores etiam Italica. Iussu Augustissimae Imperatricis et Reginae Apostolicae nunc secundis curis recognitum et auctum. Vienna, Typis Iosephi Nobilis de Kurzböck.

Juynboll, G[autier] H.A. (2007) Encyclopedia of Canonical Hadìth. Leiden-Boston. Lerchundi, José [Antonio Ramón] (1892) Vocabulario español-arábigo del dialecto de Marruecos, con gran número de voces usadas en Oriente y en la Argelia. Tánger, Imprenta de la Misión católico-española. 
Lombard, A[lf] (1936) Die Bedeutungsentwicklung zweier ibero-romanischer Verba. Zeitschrift für romanische Philologie 56, 637-43.

Marçais, William (1902) Le dialecte arabe parlé à Tlemcen. Paris.

Marçais, William (1906) L'euphémisme et l'antiphrase dans les dialectes arabes d'Algérie, in Carl Bezold (hrsg), Orientalische Studien Theodor Nöldeke zum siebzigsten Geburtstag (2 März 19o6) gewidmet, I, 425-38. Gieszen.

Martin, Laura (1986) "Eskimo Words for Snow": a Case Study in the Genesis and Decay of an Anthropological Example. American Anthropologist 88, 418-23.

Meninski (1680) Thesaurus linguarum orientalium Turcicce, Arabicce, Persicce, Præcipuas earum opes à Turcis peculiariter usurpatas continens, nimirum Lexicon TurcicoArabico-Persicum [...], \& Grammaticam Turcicam. [...] Operâ, typis, \& Sumptibus Francisci à Mesgnien Meninski. Viennæ (Austriæ). In quinque Tomos distributus.

Merrick, G[George Charleton] (1905) Hausa Proverbs. London.

Monchicourt, Ch[arles] (1908) Répugnance ou respect realatifs à certaines paroles ou à certains animaux. Revue Tunisienne $15,5^{-21}$.

Moscoso García, Francisco (2005) Diccionario español-árabe maroquí. Dirección General de Coordinación de Políticas Migratorias-Consejería de GobernaciónJunta de Andalucía.

Pullum, Geoffrey K. (1991a) The Great Eskimo Vocabulary Hoax and Other Irreverent Essays on the Study of Language. Chicago-London.

Pullum, Geoffrey K. (1991b) The Great Eskimo Vocabulary Hoax, in Pullum (1991a), 159-71.

Sandfeld, Kr[istian] (1930) Linguistique balcanique. Problèmes et résultats (Collection linguistique publiée par la Société de linguistique de Paris 31). Paris.

Scancarelli, Janine (1994) Another Look at a "Primitive Language". International Journal of American Linguistics 6o, 149-6o.

Sher 'Alī (2004) The Holy Qur'ān. Arabic Text and English Translation. Translated by Maulawī Sher 'Alī, with an alternative translation of, and footnotes to, some of the verses, by Mirzā Ṭahiir Ahmād. Islamabad (PK)—Tilford (UK). [First edn. 1955].

Shivtiel, Avihai (2009) Taboo, in Kees Versteegh (2005-2009), Encyclopedia of Arabic Language and Linguistics, 5 voll. (general ed. Kees Versteegh, associate eds. Mushira Eid, Alaa Elgibali, Manfred Woidich, Andrzej Zaborski), IV, 416-21. Leiden-Boston. Shivtiel, Avihai (2010) Language and Mentality: Politeness, Courtesies and Gestures in Palestinian Arabic, in Lutz Edzard, Stephan Guth (eds.), Verbal Festivity in Arabic and other Semitic Languages. Proceedings of the Workshop at the Universitätsclub Bonn on January 16, 2009, 31-42. Wiesbaden.

Skeat, Walter William (1900) Malay Magic, Being an Introduction to the Foklore and Popular Religion of the Malay Peninsula, with a Preface by Charles Otto Blagden. London. 
Trachtenberg, Joshua (1939) Jewish Magic and Superstition. A Study in Folk Religion, New York. [Publicly available e-text, scanned, proofed and formatted in 2008 by John Bruno Hare: http://www.sacred-texts.com/jud/jms/jmsoo.htm].

Traini, Renato (1993) Vocabolario arabo-italiano (con la collaborazione di Maria Nallino). Roma.

Watson, Janet C.E., Abdullah Musallam al-Mahri (2017) Language and Nature in Dhofar, in Simone Bettega, Fabio Gasparini (eds.), Linguistic Studies in the Arabian Gulf (Special issue of QuadRi-Quaderni di Ricognizioni 7/2017), 87-103. Torino.

Weber, Robert, Roger Gryson (2007) Biblia sacra ex vulgatam versionem, adiuvantibus B. Fischer, I. Gribomont, H.F.D. Sparks, W. Thiele, recensuit et breve apparatu critico instruxit Robert Weber. Editionem quintam emendatam retractatam preparavit Roger Gryson. Stuttgart. [Editio prima, 1969].

Wehr (1976) The Hans Wehr Dictionary of Modern Written Arabic, edited by J. M[ilton] Cowan, Ithaca (NT). [Based on the 3rd edn., Wiesbaden 1971 (1st edn. 1961)].

Westermarck, Edward (1914) Marriage Ceremonies in Morocco. London.

Westermarck, Edward (1926) Ritual and Belief in Morocco, 2 vols. London.

Westermarck, Edward (1930) Wit and Wisdom in Morocco. A Study of Native Proverbs, with the assistance of Shereef 'Abd-es-Salam el-Baqqali. London.

Wilton, David (2004) Word Myths. Debunking Linguistic Urban Legends (illustrated by Ivan Brunetti). Oxford UP. 